\title{
Vitesse d'émission des feuilles des brins maîtres d'une orge de printemps cultivée à plusieurs altitudes : différences liées au type de tallage et aux milieux
}

\author{
M Lafarge 1 \\ avec la collaboration technique de $E$ Albaret ${ }^{2}, V$ André ${ }^{1 *}, B_{\text {Carette }}{ }^{1 *}$, \\ D Ezard 1, J Hanoteaux 1, A Mante 2, C Pravin 1* \\ 1 INRA, station d'agronomie, 12, avenue du Brézet, 63039 Clermont-Ferrand; \\ 2 INRA, domaine de Marcenat, La Borie, 15330 Marcenat, France
}

(Reçu le 8 juillet 1991; accepté le 2 septembre 1992)

\begin{abstract}
Résumé - Cette ètude compare des cultures d'un même lot de semences mises en place à $320 \mathrm{~m}, 880 \mathrm{~m}$ et 1120 $\mathrm{m}$ d'altitude en sortie d'hiver, et après le réchauffement printanier à $880 \mathrm{~m}$. Des échantillons des peuplements obtenus sont prélevés après le début de montaison, vers l'arrêt du tallage. Les plantes sans talle, parfois très fréquentes en montagne, émettent leurs feuilles plus lentement que celles qui tallent à partir du nœud de 1re feuille (type T1+). Pour un même type de tallage et dans une situation culturale donnée, l'émission est plus rapide en peuplements clairs bien alimentés en azote. Les plantes de type T1+ ayant poussé en peuplements clairs bien alimentés en azote présentant des phyllochrones différents entre situations culturales, en fonction des conditions climatiques initiales. Les facteurs autres que climatiques pourraient aussi agir par conditionnement initial des plantules.
\end{abstract}

Hordeum vulgare $\mathrm{L}=$ orge $/$ phyllochrone / altitude $/$ type de tallage $/$ milieu

Summary - Rates of leaf emergence on main stems of spring barley grown at several elevations : differences associated with types of tillering and environmental effects. Our experimental design compared several crops sown in the highlands of the French Massif Central to a control sown at a low elevation, all of them using the same seed lot (cultivar and origin). The crops were sown in late winter at each elevation (320, 880, $1120 \mathrm{~m}$ ) and again somewhat later at $880 \mathrm{~m}$. Plant emergence was relatively homogeneous (table 1). Sample plants were harvested for investigation following the onset of shooting (table II). They were subjected to well differentiated climatic conditions (fig 1, table VI), and their mineral levels already reflected the experimental conditions (table III).

Plants without tillers (type "ST") were numerous at times in the high elevation stands (fig 2). They put forth leaves at a slower rate than plants showing the usual type of tillering from the first leave node onwards (type T1+; fig 2; table V). In a given growing location plants belonging to the same tillering type put forth leaves more rapidly in thin stands that were well supplied with nitrogen (table $V I$ ). The phyllochron of type $T 1+$ plants was variable among similar thin stands well supplied with nitrogen but grown at different locations (table VII). These phyllocrons could be associated with the climatic criteria reported in the literature (table VIII), according to the means by which time is measured (fig 3).

The late of foliar emergence was modified according to tillering type, mineral nutrition, stand density, and climate. All these fators seem to act through an initial conditioning of the seedlings.

Hordeum vulgare L / leaf emergence rate / altitude / type of tillering / environment

* Stagiaire INAPG 


\section{INTRODUCTION}

La structure d'un peuplement végétal contrôle ses relations trophiques avec le milieu (MasleMeynard et Sébillotte, 1981a) et détermine de ce fait l'élaboration de la production (Duru et Langlet, 1988). Certains résultats suggèrent que cette structure se forme et évolue autrement en montagne qu'en plaine. Par exemple, le tallage herbacé et le tallage épi moyens de culture d'une même variété de céréale sont différemment affectés par l'altitude (Kobold, 1974; Midmore et al, 1984). La structure d'un peuplement céréalier résulte de la distribution de plantes différant notamment par le nombre et la position des talles présentes (Cannell, 1969). MasleMeynard et Sébillotte (1981a) proposent de classer ces plantes en quelques types morphologiques définis par le rang de la feuille qui axille la plus ancienne talle du pied, quel que soit le nombre de talles qu'il porte. On appellera «type de tallage" ce critère de classement.

L'émission des feuilles sur chaque plantule construit la structure d'un jeune peuplement en rythmant l'apparition des talles (Masle-Meynard et Sébillotte, 1981b) et des racines (Klepper et al, 1984), et en contribuant à la croissance de la surface foliaire de chaque tige (Gosse et al, 1986). Sur une échelle de temps thermique (somme de degrés-jours), le rythme d'émission des feuilles d'un brin maitre est suffisamment stable (Baker et al, 1980, 1986; Kirby et al, 1982) pour être pris comme caractère morphogénétique individuel (Malvoisin, 1984). On le représente souvent par son inverse, le phyllochrone, c'est-à-dire le temps écoulé entre l'apparition de 2 feuilles successives (Baker et al, 1986). Dans les études où l'apparition des feuilles est repérée par l'émergence de la pointe du limbe ou quantifiée selon l'échelle de Haun (1973), on observe un changement de rythme vers l'initiation florale (Baker et al, 1986; certains résultats de Hay et Delécolle, 1989; Boone et al, 1990; Cao et Moss, 1991; Ntonga, 1992). Cette rupture de pente ne se manifeste plus quand on tient compte de l'apparition des ligules (Malvoisin, 1984; Hay et Kemp, 1990; Ntonga, 1992).

Dans un même peuplement on observe des plantes à phyllochrones différents (Malvoisin, 1984; Hay et Delécolle, 1989). Les types définis par Masle-Meynard et Sébillotte (1981a) chez le blé se distinguent également par leur phyllochrone. Entre cultures d'une même variété, la vitesse moyenne d'émission des feuilles change entre sites et dates de semis (Kirby et al, 1985) principalement à cause de la photopériode (Baker et al, 1980; Kirby et al, 1982; Cao et Moss, 1989; Masle et al, 1989). Elle change aussi avec la densité de population au champ (Kirby et Faris, 1970) et selon la richesse en azote de la solution nutritive (Dale et Wilson, 1978), au moins chez l'orge de printemps. La comparaison de phyllochrones moyens entre peuplements pose le problème de leur composition en plantes "lentes" et "rapides".

Des semis de printemps étagés en altitude créent des conditions assez contrastées de photopériode et de température (Lafarge, 1991) dans une gamme différente des comparaisons souvent pratiquées entre semis d'automne, hiver et printemps en plaine (Kirby et al, 1982; Hay et Delécolle, 1989). Dans la présente étude, on recherche sur orge si les types de plantes définis sur blé par Masle-Meynard et Sébillotte (1981a) manifestent des différences de phyllochrone et si elles sont à peu près stables entre milieux. On analyse ensuite l'effet des conditions de milieu sur la vitesse d'émission des feuilles pour des plantes présentant un même type de tallage.

\section{MATÉRIEL, MÉTHODES ET CONDITIONS}

\section{Dispositif d'étude}

Le dispositif utilisé fait partie d'un ensemble également destiné à l'étude du tallage, de la fertilité d'épi et de la croissance (Lafarge, 1991). Il associe 3 sites en Auvergne : Malintrat, à $320 \mathrm{~m}$, en sol argilo-calcaire dans la plaine céréalière de Clermont, Saint-Genès, à 880 $\mathrm{m}$, en sol brun acide sur le plateau herbager demimontagnard, et enfin Landeyrat, à $1120 \mathrm{~m}$, sur sol andique en zone d'estive. Pour la présente étude, ce dispositif comporte 4 traitements élémentaires mis en place dans 5 situations culturales constituées par:

- un semis de sortie d'hiver («semis normal»), en sol juste réchauffé (environ $6^{\circ} \mathrm{C}$ ) dans chaque site,

- le 24 février à Malintrat,

- le 3 avril à Saint-Genès,

- le 3 mai à Landeyrat.

entre ces 3 situations, les longueurs de jour en début de végétation croissent avec la date de semis (fig 1);

- un semis de mi-mai à Saint-Genès, souvent pratiqué par les éleveurs des environs; il réalise un début de végétation à peu près contemporain de celui de Landeyrat, en jours "longs" (environ $15 \mathrm{~h}$ ), mais à des températures plus chaudes $\left(9^{\circ} \mathrm{C}\right.$ dans le sol pendant la germination); ce semis tardif concerne 2 situations culturales différentes par l'état structural du sol :

- la première a été semée le 17 mai sur la même préparation soignée que le semis normal, 

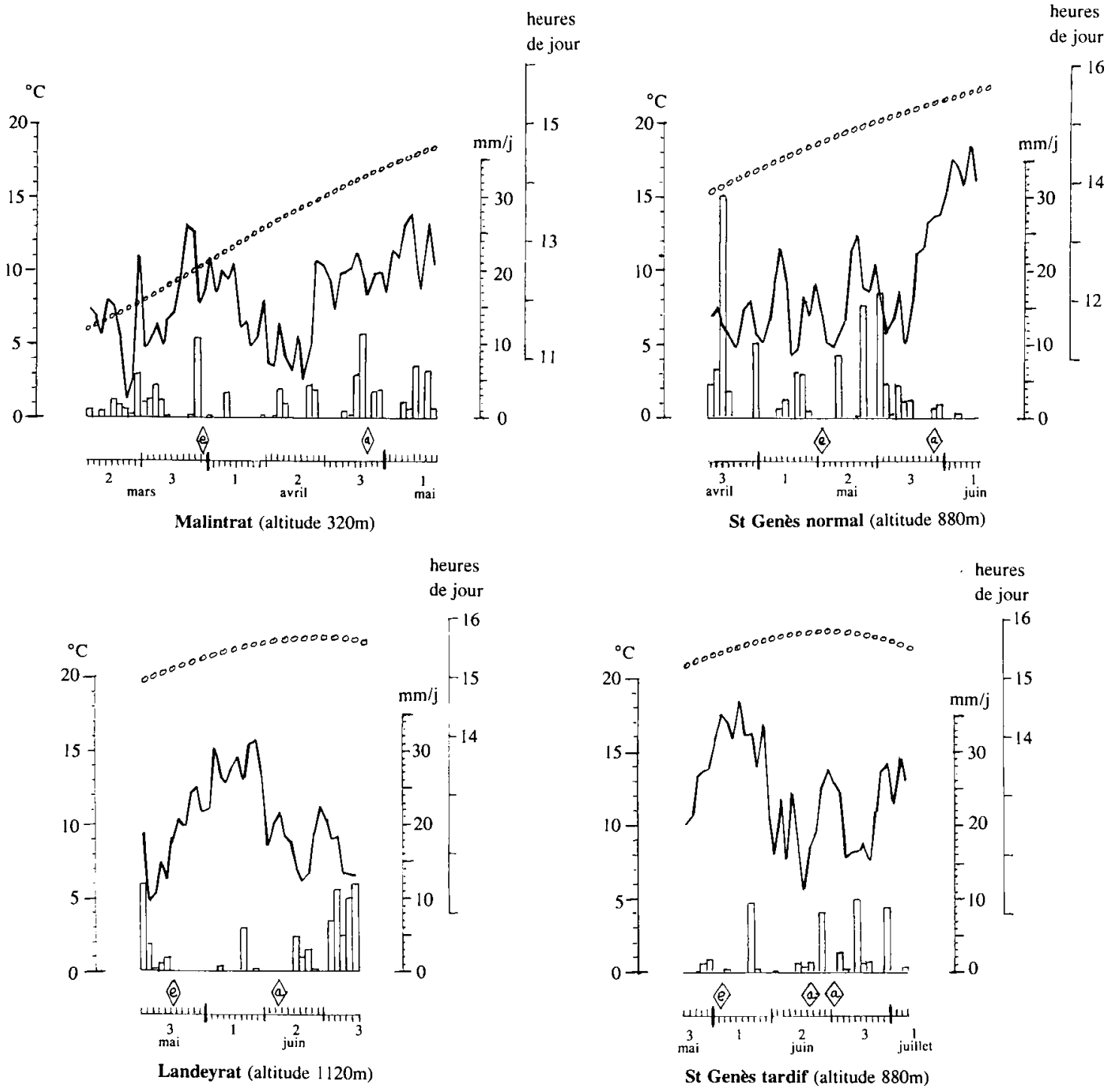

Fig 1. Longueurs du jour, températures moyennes et précipitations quotidiennes, de la levée au prélèvement étudié (année 1978).

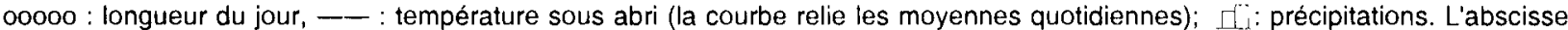
est graduée en jours, avec identification du mois et de la décade. Sur l'abscisse sont repérées les dates de l'épandage d'engrais (e) et du prélèvement pour analyse d'azote (a).

- la seconde le 20 mai sur une préparation superficielle hâtive laissant le sol compact en-dessous.

Les 4 traitements élémentaires, $\mathrm{BO}, \mathrm{BN}, \mathrm{FO}$ et $\mathrm{FN}$, proviennent du croisement de traitements expérimentaux de densité de semis et d'alimentation azotée, dont les modalités sont les suivantes:

- une basse densité de semis ("B" = 150 grains $/ \mathrm{m}^{2}$ ) identique partout, et une forte densité ( $F ")$ de 300 grains $/ \mathrm{m}^{2}$ à Malintrat, 500 à Saint-Genès "semis normal" et de 650 à Landeyrat et Saint-Genès "tardif» (progression visant l'obtention d'un rendement maximal dans chaque situation);

- un apport d'azote après levée, modulé selon le reliquat post-hivernal, définit la modalite "N". La dose est de $80 \mathrm{~kg} / \mathrm{ha}$ à Landeyrat et de 50 dans les autres situations. L'autre modalité, "O» est l'absence d'apports d'engrais azoté.
L'orge de printemps utilisée est la variété à 2 rangs Bérénice. Toute la semence provient d'un même lot de prébase. Dans chaque parcelle, les lignes de semis sont espacées de $25 \mathrm{~cm}$ pour permettre des binages en cas de désherbage insuffisant. II y a 6 blocs en semis normaux et 3 dans chaque situation de Saint-Genès tardif. On identifie ces 2 situations par leurs numéros de blocs : “1, 2, 3" pour le premier semis sur préparation soignée, et « $7,8,9$ » pour le second semis sur préparation superficielle hâtive. On appelle "culture élémentaire" la cellule du dispositif constituée par un traitement élémentaire dans une situation culturale. À Landeyrat, les fortes densités ont été répétées de part et d'autre du dispositif de base pour permettre une comparaison entre présence et absence de traitement phytosanitaire. Celui-ci n'ayant été appliqué qu'après le prélèvement étudié ici, on dispose de 12 parcelles par culture élémentaire en 
fortes densités à Landeyrat. À Saint-Genès et Landeyrat, les blocs sont disposés en fonction d'une légère pente du terrain, absente à Malintrat où I'on a seulement tenu compte des facilités de circulation.

\section{Mesures et méthodes}

Les résultats proviennent d'un prélèvement destructif destiné par ailleurs à l'étude de tallage et effectué après le début de montaison, quand la raréfaction des talles à 1 feuille a signalé l'arrêt du tallage (MasleMeynard, 1982). Une placette de $0,26 \mathrm{~m}^{2}(0,35 \mathrm{~m}$ de ligne sur les 3 lignes centrales) a été prélevée par arrachage dans chaque parcelle, au même moment sur tous les traitements d'une mème situation culturale. A Landeyrat, on a évité les zones où une intoxication aluminique s'était manifestée. Les 25 plantes à dépouiller par placette ont été échantillonnées par tirage systématique : en basses densités, 1 plante sur 2 , d'abord sur le prélèvement complet puis sur le reste. En fortes densités, on a tiré de la même manière 1 plante sur 3. Les plantes dont le brin maître avait été détruit par un asticot d'Oscinella frit $L$ ont èté exclues de l'échantillon étudié ici.

Les feuilles apparentes sur les brins maitres ont été simplement comptées, sans notation décimale d'émergence du dernier limbe. Un limbe encore enroulé n'a cependant été compté que si la ligule de la feuille précédente était visible. L'âge foliaire d'un brin maître à $n$ feuilles est compris entre $(n-1)+0,1$ et $(n-$ 1) $+0,9$. L'âge foliaire moyen d'une population de plantes ayant en moyenne $N$ feuilles est alors $N-0,5$.

Pour estimer le niveau de nutrition azotée effectivement réalisé dans la période qui nous occupe, le modèle de Greenwood et al (1986) a été appliqué à l'analyse d'un prélèvement du couvert effectué vers le stade "épi à $1 \mathrm{~cm}$ ", 1-2 semaines avant le prélèvement étudié.

Le tableau I donne les éléments de datation de la levée. La dispersion intrapeuplement peut être appréciée rétrospectivement, sur la base de la correspondance proposée par Haun (1973) entre âge et niveau de déroulement de la $1^{r e}$ feuille. Cette information peut suppléer l'absence d'une observation de début ou de fin d'émergence. La dispersion est plus forte à Landeyrat et Saint-Genès tardif «7, 8, 9» qu'ailleurs, mais sans bimodalité. La date de levée retenue correspond à plus de $50 \%$ de plantes levées. Pour les comparaisons entre situations, le premier jour pris en compte pour le cumul du temps est le lendemain de cette "date de levée" : le percement du coléoptile a dû s'y produire sur la majorité des plantes (tableau I). Le tableau II donne les durées ainsi écoulées jusqu'au jour du prélèvement, en somme des degrés jours en base $0{ }^{\circ} \mathrm{C}$ (Gallagher et al, 1983) et en "temps photothermique" selon Masle et al (1989) : il s'agit d'une somme des degrés jours en phase diurne multipliée par la photopériode contemporaine.

Les résultats ont été examinés statistiquement par analyse de variance. Pour pouvoir traiter chaque

Tableau I. Datation de la levée.

\begin{tabular}{|c|c|c|c|c|c|}
\hline & \multirow[t]{2}{*}{ Malintrat } & \multirow{2}{*}{$\begin{array}{l}\text { Saint-Genès } \\
\text { normal }\end{array}$} & \multirow[t]{2}{*}{ Landeyrat } & \multicolumn{2}{|c|}{ Saint-Genès tardif } \\
\hline & & & & $" 1,2,3 »$ & $« 7,8,9 »$ \\
\hline $\begin{array}{l}\text { Début de levée } \\
\text { observé le }\end{array}$ & 11 mars & 21 avril & 18 mai & 25 mai & $?$ \\
\hline $\begin{array}{l}\text { Levée totale } \\
\text { observée le }\end{array}$ & 13 mars & 24 avril & 22 mai & $?$ & 30 mai \\
\hline $\begin{array}{l}\text { Observation } \\
\text { de la fre feuille } \\
\text { date } \\
\text { stade de Haun } 1\end{array}$ & $\begin{array}{l}16 \text { mars } \\
\text { env } 0,6\end{array}$ & $\begin{array}{l}28 \text { avril } \\
\text { env } 0,6\end{array}$ & $\begin{array}{l}26 \text { mai } \\
0,2-1,0 \\
\text { majorité vers } 0,6\end{array}$ & $\begin{array}{l}30 \text { mai } \\
\text { env } 0,8\end{array}$ & $\begin{array}{l}30 \text { mai } \\
\text { de «avant } 0,0 " 1 \\
\text { à } 0,4\end{array}$ \\
\hline $\begin{array}{l}\text { Date retenue } \\
\text { pour la levée }\end{array}$ & 12 mars & 23 avril & 21 mai & 27 mai & 29 mai \\
\hline $\begin{array}{l}\text { Temps thermique } \\
\text { du jour suivant la } \\
\text { date de levée au } \\
\text { jour d'observation de } \\
\text { la } 1^{\text {re }} \text { feuille (inclus) }\end{array}$ & $28 \mathrm{dj}$ & $32 \mathrm{dj}$ & $32 \mathrm{dj}$ & $39 \mathrm{dj}$ & $14 d j$ \\
\hline
\end{tabular}

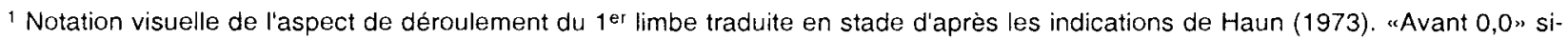
gnifie coléoptile non percé
} 
Tableau II. Durées entre la levée et le prélèvement. La période prise en compte s'étend du lendemain de la date de levée au jour du prélèvement inclus.

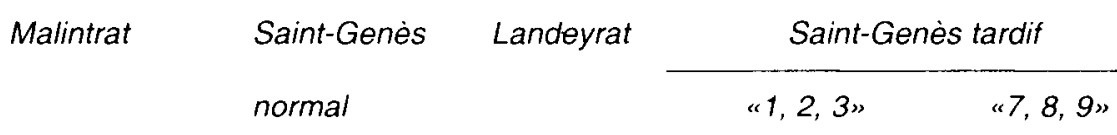

$\begin{array}{llllll}\begin{array}{l}\text { Date du } \\ \text { prélèvement }\end{array} & 9 \text { mai } & 6 \text { juin } & 26 \text { juin } & 3 \text { juillet } & 3 \text { juillet } \\ \begin{array}{l}\text { Nombre de jours } \\ \begin{array}{l}\text { Durée } \\ \text { thermique }{ }^{1}\end{array}\end{array} & 58 & 44 & 36 & 37 & 35 \\ \begin{array}{l}\text { Durée } \\ \text { photothermique }{ }^{2}\end{array} & 301 \mathrm{dj} & 407 \mathrm{dj} & 359 \mathrm{dj} & 458 \mathrm{dj} & 433 \mathrm{dj} \\ \end{array}$

1 Somme des degrés jours en base $0^{\circ} \mathrm{C}$ (Gallagher et al, 1983) obtenus à partir des températures moyennes de l'air sous abri, calculées quotidiennement par $\left(T_{\operatorname{mini}}+T_{\text {maxi }}\right) / 2 .{ }^{2}$ Notion et calcul d'après Masle et al (1989) : somme sur la période des valeurs (température diurne $x$ fraction de $24 \mathrm{~h}$ en phase éclairée). La température moyenne diurne est approchée par la formule $: T_{\text {mini }}+0,65$ $\left(T_{\operatorname{maxi}}-T_{\text {mini }}\right)$.

plante dépouillée comme un individu malgré les différences d'effectifs dues aux nombres de parcelles ou aux distributions entre sous-populations retenues, il faut disposer d'une méthode bien adaptée aux dispositifs non orthogonaux. Nous avons utilisé celle que propose le logiciel "Amance 81" (Bachacou et al, 1981); les comparaisons de moyennes sont faites par une méthode de "T corrigé".

\section{Nutrition azotée, conditions climatiques et fermeture du couvert}

Les analyses (tableau III) montrent que les cultures fertilisées ont différemment absorbé l'engrais selon les situations culturales. En particulier, on n'atteint pas à Landeyrat le niveau de nutrition considéré

Tableau III. Teneurs en azote des parties aériennes. L'épandage d'engrais et le prélèvement pour analyse sont datés sur la fig 1; ce dernier précède le prélèvement étudié d'environ 110 dj sur les semis normaux, et 160 dj sur St-Genès tardif.

\begin{tabular}{|c|c|c|c|c|c|}
\hline $\begin{array}{l}\text { Situation } \\
\text { culturale }\end{array}$ & Teneurs & $B O$ & $B N$ & FO & $F N$ \\
\hline Malintrat & $\begin{array}{l}N \% \text { poids sec } \\
N \% \text { Ncrit } 1\end{array}$ & $\begin{array}{l}4,2 \\
88 \%\end{array}$ & $\begin{array}{l}4,7 \\
97 \%\end{array}$ & $\begin{array}{l}3,8 \\
84 \%\end{array}$ & $\begin{array}{l}4,4 \\
100 \%\end{array}$ \\
\hline $\begin{array}{l}\text { Saint-Genès } \\
\text { normal }\end{array}$ & $\begin{array}{l}N \% \text { poids sec } \\
N \% \text { Ncrit } 1\end{array}$ & $\begin{array}{l}4,2 \\
84 \%\end{array}$ & $\begin{array}{l}5,1 \\
104 \%\end{array}$ & $\begin{array}{l}3,3 \\
73 \%\end{array}$ & $\begin{array}{l}4,5 \\
101 \%\end{array}$ \\
\hline Landeyrat & $\begin{array}{l}\mathrm{N} \% \text { poids sec } \\
\mathrm{N} \% \text { Ncrit } 1\end{array}$ & $\begin{array}{l}4,5 \\
84 \%\end{array}$ & $\begin{array}{l}5,0 \\
95 \%\end{array}$ & $\begin{array}{l}3,7 \\
75 \%\end{array}$ & $\begin{array}{l}4,3 \\
85 \%\end{array}$ \\
\hline $\begin{array}{l}\text { Saint-Genès } \\
\text { tardif } \\
\text { (situations confondues) }\end{array}$ & $\begin{array}{l}N \% \text { poids sec } \\
N \% \text { Ncrit }{ }^{1}\end{array}$ & $\begin{array}{l}5,1 \\
101 \%\end{array}$ & $\begin{array}{l}5,3 \\
106 \%\end{array}$ & $\begin{array}{l}4,2 \\
90 \%\end{array}$ & $\begin{array}{l}4,7 \\
102 \%\end{array}$ \\
\hline
\end{tabular}

${ }^{1} \mathrm{~N} \%$ Ncrit. : Teneur en azote rapportée à la "teneur critique" de Greenwood et al (1986) considérée comme non limitante pour les gains de poids sec dans leur modèle de dilution. 
comme non limitant pour la croissance. Ces comportements peuvent être rapportés aux bilans hydriques (tableau IV) : Landeyrat, très déficitaire entre épandage et mesure et n'ayant connu qu'une pluie faiblement incorporante, s'oppose à Saint-Genès normal, excédentaire dans cette période et dont le sol venait de subir des pluies lessivantes. L'écart de nutrition azotée entre traitements fertilisés et non fertilisés est maximal à Saint-Genès normal (tableau III).

La figure 1 montre l'évolution de la longueur du jour, la température de l'air et les pluies quotidiennes entre la levée et le prélèvement étudié, pour les différentes situations culturales. La sécheresse est principalement due à la période chaude de début juin. Ultérieurement, les pluies répétées de Landeyrat tendent à équilibrer le bilan depuis levée, alors que SaintGenès tardif, qui poursuit une sécheresse modérée apparaît nettement déficitaire sur l'ensemble de la période étudiée (tableau IV). La pluie incorporant l'engrais au sol est survenue quand les plantes avaient 2-3 feuilles en montagne et 3-4 à Malintrat.

Le jour du prélèvement, le couvert végétal n'est régulièrement fermé qu'en fortes densités à Malintrat, et le sol de l'interligne reste bien visible en basses densités à Landeyrat et Saint-Genès tardif « $7,8,9 »$.

\section{Distribution des types de plantes dans les peuplements}

Les types de plantes sont définis comme chez Masle-Meynard et Sébillotte (1981a), mais codés plus explicitement. On distingue les plantes tallant dès la talle de coléoptile (notées $\mathrm{TC}_{+}$), celles qui commencent par les talles de 1 re $\mathrm{T} 1+)$, de $2^{e}(\mathrm{~T} 2+)$ ou de $3^{e}$ feuille $(\mathrm{T} 3+)$, et celles qui ne portent aucune talle (ST).
L'âge de certaines plantes au moment du prélèvement nous interdit de distinguer sans ambiguïté les types St et T3+ sur nos prélèvements. Si l'on admet le modèle de tallage de Masle-Meynard et Sébilotte (1981b), les plantes sans talle n'ayant que 5 feuilles au moment de l'observation peuvent encore théoriquement passer dans les $\mathrm{T} 3+$, l'émission de $\mathrm{T}_{3}$ étant contemporaine de celle de la $6^{e}$ feuille dans le modèle. Ce problème et l'effectif partout faible des T3 (au maximum $4 \%$ ) nous ont amené dans cette étude à regrouper les ST et les T3+ en une seule catégorie. La figure 2 montre les contributions des différents types de tallage aux peuplements dans chaque culture élémentaire.

\section{RÉSULTATS}

\section{Différences liées au type de tallage}

La figure 2 suggère et l'analyse de variance montre l'existence de différences de nombre de feuilles entre types de tallage dans une situation culturale donnée. A Malintrat par exemple, les plantes ayant des talles à partir du nœud de coléoptile ont significativement plus de feuilles que celles qui ne tallent qu'à partir des nœuds de $1^{\text {re }}$ ou $2^{\mathrm{e}}$ feuille (respectivement $8,1,7,6$ et 6,9 ).

En montagne, les effectifs du groupe ST et $\mathrm{T} 3+$ permettent de le comparer aux $\mathrm{T} 1+$ dans chacune des conditions créées par 11 cultures élémentaires (tableau $V$ ). Dans la plupart des cas, les plantes de type $\mathrm{T} 1+$ ont émis au moment du prélèvement très significativement plus de feuilles que celles de l'autre catégorie. Même

Tableau IV. Bilans hydriques en relation avec la mise à disposition de l'engrais.

\begin{tabular}{|c|c|c|c|c|}
\hline \multirow[t]{2}{*}{$\begin{array}{l}\text { Situation } \\
\text { culturale }\end{array}$} & \multirow{2}{*}{$\begin{array}{l}\text { de la levée } \\
\text { à l'apport } \\
\text { d'engrais } \\
\text { P-ETM } \\
(\mathrm{mm})\end{array}$} & \multicolumn{2}{|c|}{$\begin{array}{c}\text { de l'apport d'engrais } \\
\text { a la mesure } \\
\text { des teneurs } \\
\text { en azote }\end{array}$} & \multirow{2}{*}{$\begin{array}{l}\text { de la mesure } \\
\text { des teneurs } \\
\text { au prélèvement } \\
\text { étudié } \\
\text { P-ETM } \\
(\mathrm{mm})\end{array}$} \\
\hline & & $\begin{array}{l}\text { pluie max } \\
\text { en } 48 \mathrm{~h}\end{array}$ & $\begin{array}{l}\text { P-ETM } \\
(\mathrm{mm})\end{array}$ & \\
\hline Malintrat & +4 & $17 \mathrm{~mm}$ & -17 & +5 \\
\hline Saint-Genès normal & +54 & $21 \mathrm{~mm}$ & +24 & -20 \\
\hline Landeyrat & -4 & $6 \mathrm{~mm}$ & -39 & +30 \\
\hline Saint-Genès tardif & -9 & $10 \mathrm{~mm}$ & -20 & -12 \\
\hline
\end{tabular}



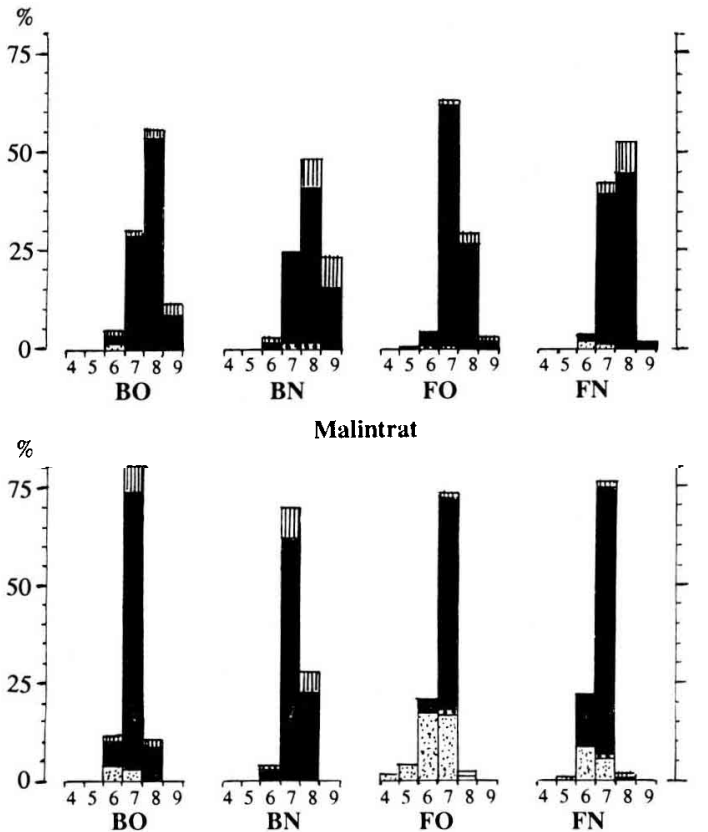

St Genès normal
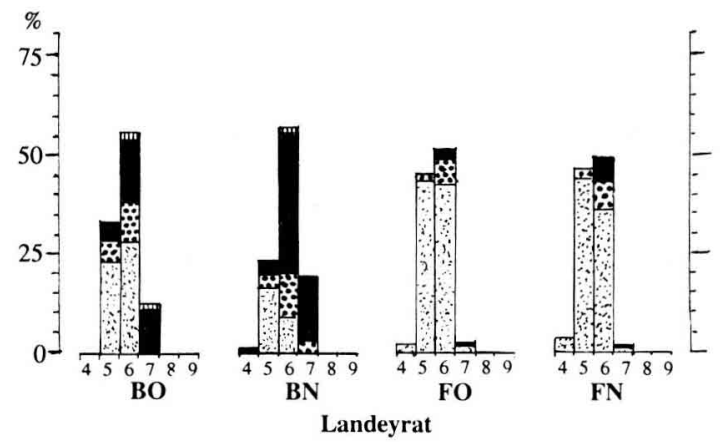

quand le terrain influence le nombre de feuilles émises tous types de plantes confondus (effet bloc), il ne modifie pas la différence entre types (interaction toujours non significative). Cette différence doit alors exister entre plantes cohabitant au sein du peuplement d'une même placette comme au niveau de l'ensemble de l'échantillon analysé.

\section{Effet des traitements et du terrain sur des plantes de même type (tableau VI)}

Les résultats précédents confirment la nécessité d'étudier l'effet des conditions induites par les traitements expérimentaux sur des populations d'un même type de tallage. A Saint-Genès et Malintrat sur les $\mathrm{T} 1+$, les conditions "BN" permettent une émission plus rapide des feuilles que les «FO». A Landeyrat, il n'y a aucune différence, que l'analyse porte sur les $\mathrm{T} 1+$ ou sur le groupe

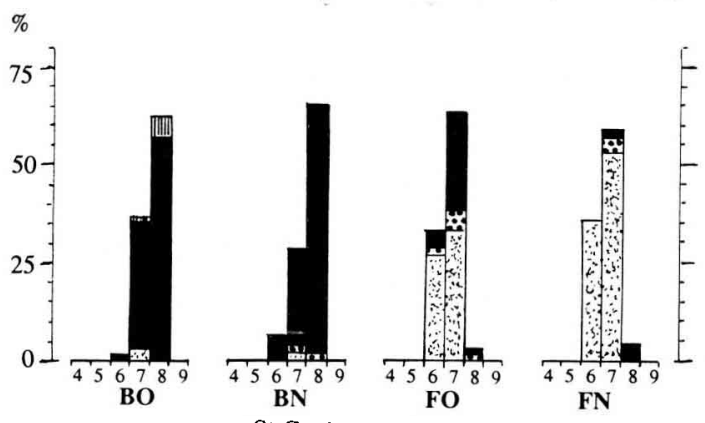

St Genès tardif "1:,2,3"
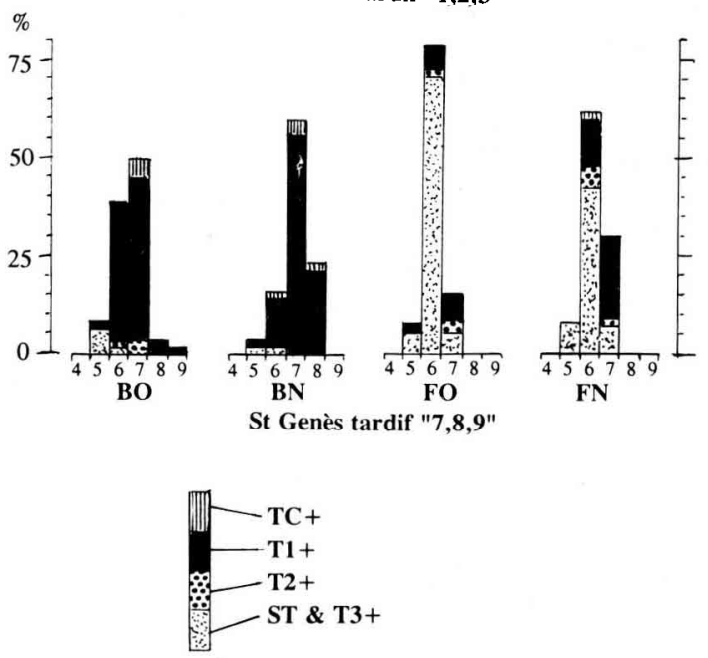

Fig 2. Distribution des plantes par type de tallage et par nombre de feuilles sur le brin maître. En abscisse : nombre de feuilles sur le brin maître. Sur les histogrammes, les trames distinguent les types de tallage (voir texte).

ST et T3+ Le terrain modifie la vitesse d'émission des feuilles et influence la réponse aux facteurs expérimentés, sauf à Saint-Genès tardif (où le nombre de blocs est faible). Les blocs significativement différents n'ont cependant pas une disposition particulière, même là où une pente pouvait créer un gradient (Landeyrat et Saint-Genès).

\section{Comparaison des phyllochrones entre situations culturales}

L'influence de la situation culturale est examinée sur des plantes de même type, les $\mathrm{T} 1+$, et dans les mêmes conditions de semis clair. On calcule leur phyllochrone moyen sur la période (tableau VII) en divisant le temps thermique ou photothermique (tableau II) par leur âge foliaire.

Dans les conditions les plus semblables d'alimentation azotée $(\mathrm{BN})$, le temps photothermique 
Tableau V. Nombres de feuilles émises au prélèvement en fonction du type de tallage des plantes. Les analyses n'ont été effectuées que dans les traitements où l'on disposait d'au moins 5 plantes de chaque type.

\begin{tabular}{|c|c|c|c|c|c|c|c|c|}
\hline \multirow{2}{*}{$\begin{array}{l}\text { Situation } \\
\text { culturale }\end{array}$} & \multirow[t]{2}{*}{ Traitement } & \multicolumn{2}{|c|}{ Effectifs } & \multirow{2}{*}{$\begin{array}{l}\text { Facteur } \\
F\end{array}$} & \multirow{2}{*}{$\begin{array}{l}\text { Type de tallage } \\
\text { Moyennes } \\
\text { ST \& T1+ } \\
\text { T3+ }\end{array}$} & \multirow{2}{*}{$\begin{array}{l}F \\
\text { bloc }\end{array}$} & \multirow{2}{*}{$\begin{array}{l}F \\
\text { inter- } \\
\text { action }\end{array}$} & \multirow{2}{*}{$\begin{array}{l}\text { CV } \\
\text { résiduels } \\
\text { (\%) }\end{array}$} \\
\hline & & $\begin{array}{l}\text { ST \& } \\
\text { T3+ }\end{array}$ & $T 1+$ & & & & & \\
\hline \multirow{3}{*}{$\begin{array}{l}\text { Saint-Genès } \\
\text { normal }\end{array}$} & BO & 9 & 113 & $12,4^{\star *}$ & $6,4<7,0^{* *}$ & $4,8^{\star \star}$ & $0,6 \mathrm{~ns}$ & 6 \\
\hline & FO & 59 & 85 & $62,6^{\star \star}$ & $6,3<7,0^{* *}$ & $1,5 \mathrm{~ns}$ & $1,4 \mathrm{~ns}$ & 8 \\
\hline & $\mathrm{FN}$ & 22 & 120 & $28,3^{\star \star *}$ & $6,3<6,8^{\star \star}$ & $3,8^{* *}$ & $2,2 n s$ & 6 \\
\hline \multirow[t]{4}{*}{ Landeyrat } & BO & 73 & 45 & $20,2^{* *}$ & $5,5<6,2^{* *}$ & $9,6^{\star *}$ & $1,5 \mathrm{~ns}$ & 8 \\
\hline & $\mathrm{BN}$ & 29 & 64 & $11,6^{\star \star}$ & $5,3<6,2^{\star *}$ & $9,4^{\star \star}$ & $0,4 \mathrm{~ns}$ & 8 \\
\hline & FO & 261 & 9 & $21,8^{* *}$ & $5,5<6,3^{* *}$ & $8,5^{* *}$ & $1,2 \mathrm{~ns}$ & 10 \\
\hline & $\mathrm{FN}$ & 237 & 20 & $16,1^{\star \star}$ & $5,4<6,0^{\star *}$ & $10,2^{\star \star}$ & $0,4 \mathrm{~ns}$ & 10 \\
\hline Saint-Genès & & & & & & & & \\
\hline $\begin{array}{l}\text { tardif } \\
\| 1,2,3 »\end{array}$ & FO & 44 & 23 & 2, ons & $6,5=6,8$ & $0,2 n s$ & $1,1 \mathrm{~ns}$ & 9 \\
\hline Saint-Genès & BO & 5 & 54 & $18,9^{* \star}$ & $5,2<6,6^{\star \star}$ & $0,2 \mathrm{~ns}$ & $0,1 \mathrm{~ns}$ & 11 \\
\hline tardif & FO & 59 & 10 & $5,3^{*}$ & $(6,0<6,4)$ & $4,0^{*}$ & $0,1 \mathrm{~ns}$ & 7 \\
\hline$(7,8,9 »$ & $\mathrm{FN}$ & 42 & 25 & $21,9^{\star \star}$ & $6,0<6,6^{* *}$ & $0,5 \mathrm{~ns}$ & $0,9 \mathrm{~ns}$ & 8 \\
\hline
\end{tabular}

** : significatif à $1 \%$ ou mieux; " : significatif à $5 \% ;()$ : différence de moyennes significative seulement à $10 \%$.

Tableau VI. Effet des conditions induites par les traitements élémentaires sur le nombre de feuilles émises lors du prélèvement pour des plantes d'un même type de tallage à l'intérieur de chaque situation culturale.

\begin{tabular}{|c|c|c|c|c|c|}
\hline \multirow{3}{*}{$\begin{array}{l}\text { Situation } \\
\text { culturale }\end{array}$} & \multirow{3}{*}{$\begin{array}{l}\text { Catégorie } \\
\text { de plantes } \\
\text { retenue pour } \\
\text { l'analyse }\end{array}$} & \multicolumn{2}{|c|}{ Facteur traitement élémentaire } & \multirow{3}{*}{$\begin{array}{l}F \\
b l o c\end{array}$} & \multirow{3}{*}{$\begin{array}{l}F \\
\text { interaction }\end{array}$} \\
\hline & & $F$ & Nombre moyen de feuilles & & \\
\hline & & & $B O, \quad B N, F O, F N$ & & \\
\hline Malintrat & $T 1+$ & $28,3^{\star *}$ & $7,7^{a}, 7,9^{a}, 7,3^{c}, 7,5^{b}$ & $8,6^{\star \star}$ & $5,8^{\star \star}$ \\
\hline $\begin{array}{l}\text { Saint-Genès } \\
\text { normal }\end{array}$ & $\mathrm{T} 1+$ & $19,4^{\star *}$ & $7,0^{\mathrm{b}}, 7,2^{\mathrm{a}}, 7,0^{\mathrm{bc}}, 6,8^{\mathrm{c}}$ & $2,8^{*}$ & $2,1^{*}$ \\
\hline Landeyrat & $\mathrm{T} 1+$ & $2,4 \mathrm{~ns}$ & $6,2^{a}, 6,2^{a}, 6,3^{a}, 6,0^{a}$ & $5,6^{\star \star}$ & $3,9^{\star \star \star}$ \\
\hline Landeyrat & ST \& T3+ & $1,1 \mathrm{~ns}$ & $5,5^{a}, 5,3^{a}, 5,5^{a}, 5,4^{a}$ & $11,9^{\star}$ & $4,5^{\star \star}$ \\
\hline $\begin{array}{l}\text { Saint-Genès } \\
\text { tardif } \\
\text { " } 1,2,3 »\end{array}$ & $\mathrm{~T} 1+$ & $12,2^{\star \star}$ & $7,6^{a}, 7,6^{a}, 6,8^{b}, 7,7^{a b}$ & 2, ons & $2,4 \mathrm{~ns}$ \\
\hline $\begin{array}{l}\text { Saint-Genès } \\
\text { tardif } \\
« 7,8,9 »\end{array}$ & $\mathrm{~T} 1+$ & $6,9^{\star \star}$ & $6,6^{\mathrm{b}}, 7,1^{\mathrm{a}}, 6,4^{\mathrm{b}}, 6,6^{\mathrm{b}}$ & $1,2 \mathrm{~ns}$ & $0,6 \mathrm{~ns}$ \\
\hline
\end{tabular}

Les valeurs situées sur une même ligne et suivies d'une même lettre ne sont pas significativement différentes. 
Tableau VII. Phyllochrones thermiques et photothermiques moyens des plantes de type $\mathrm{T} 1+$ entre situations culturales pour un même semis clair. Les valeurs d'une même colonne suivies d'une même lettre ne sont pas significativement différentes.

\begin{tabular}{|c|c|c|c|c|}
\hline & \multicolumn{2}{|c|}{ Conditions $B N$} & \multicolumn{2}{|c|}{ Conditions $B O$} \\
\hline & $\begin{array}{c}\text { temps } \\
\text { thermique }\end{array}$ & $\begin{array}{c}\text { temps } \\
\text { photo- } \\
\text { thermique }\end{array}$ & $\begin{array}{c}\text { temps } \\
\text { thermique }\end{array}$ & $\begin{array}{l}\text { temps } \\
\text { photo- } \\
\text { thermique }\end{array}$ \\
\hline Valeur du F & $8,8^{\star \star}$ & $28,6^{\star \star}$ & $22,1^{* *}$ & $53,1^{* *}$ \\
\hline Malintrat & $63^{b}$ & $41^{a}$ & $65^{a}$ & $42^{\mathrm{a}}$ \\
\hline $\begin{array}{l}\text { St-Genès } \\
\text { normal }\end{array}$ & $61^{a}$ & $43^{b}$ & $63^{a}$ & $44^{b}$ \\
\hline Landeyrat & $64^{b c}$ & $46^{c}$ & $64^{a}$ & $46^{\mathrm{bc}}$ \\
\hline $\begin{array}{l}\text { St-Genès } \\
\text { tardif } \\
« 1,2,3 »\end{array}$ & $65^{b c}$ & $47^{c}$ & $65^{a}$ & $46^{c}$ \\
\hline $\begin{array}{l}\text { St-Genès } \\
\text { tardif } \\
« 7,8,9 »\end{array}$ & $67^{c}$ & $48^{c}$ & $72^{b}$ & $52^{d}$ \\
\hline
\end{tabular}

Remarque : La méthode de comparaison de moyennes ( $T$ corrigé) tient compte de différences éventuelles de variance; celles-ci peuvent entraîner un classement différent pour 2 moyennes numériquement égales.

permet de bien distinguer Malintrat, Saint-Genès normal, et l'ensemble des situations levées fin mai, Landeyrat et les semis tardifs de SaintGenès. L'effet du facteur "situation culturale" est renforcé en l'absence de fumure azotée ( $F$ plus élevé), à cause de l'apparition d'un fort écart entre les 2 situations de Saint-Genès tardif.

La part climatique de l'effet "situation culturale" peut être précisée en corrélant les phyllochrones obtenus en $\mathrm{BN}$ à des facteurs réputés déterminants par la littérature. II s'agit de conditions au début de la végétation : la vitesse de variation de la longueur du jour à la levée (Baker et al, 1980; Kirby et al, 1982, 1985; Ellis et Russell, 1984) ou bien le rapport "température moyenne/durée du jour" (Cao et Moss, 1989). Leurs valeurs pour nos différentes situations culturales figurent au tableau VIII. Le critère de Cao et Moss (1989) a été calculé en partant de la levée de 2 façons : sur un mois, comme indiqué - sans justifications particulières - dans l'article, et sur 70-80 degrés jours, durée couvrant le déploiement complet de la $1^{\text {re }}$ feuille et représentant des conditions plus franchement initiales. Le phyllochrone thermique ne s'ajuste linéairement qu'au critère de Cao et Moss (1989) calculé sur un mois; la régression est $Y=53,7^{\star \star}+15,2 X^{*}$ $\left(r^{2}=89 \% *, n=5\right)$. Le phyllochrone photothermique se révèle fortement lié à la variation initiale de la photopériode (fig 3).

\section{DISCUSSION}

\section{Différences intrapeuplement et effets des traitements intrasituation}

Nous retrouvons pour l'orge et dans une large gamme de milieux les différences de vitesse

Tableau VIII. Valeurs enregistrées sur notre dispositif à partir de la levée pour des variables climatiques réputées explicatives de différences de phyllochrone (voir texte).

\begin{tabular}{|c|c|c|c|}
\hline Malintrat & Saint-Genès & Landeyrat & Saint-Genès tardif \\
\hline
\end{tabular}

\begin{tabular}{|c|c|c|c|c|c|}
\hline $\begin{array}{l}\text { Variation quotidienne } \\
\text { de la longueur du jour } 1\end{array}$ & $+5,5$ & +4 & +3 & $+2,5$ & $+2,5$ \\
\hline \multicolumn{6}{|c|}{$\begin{array}{l}\text { Rapport de la température } \\
\text { moyenne quotidienne } \\
\text { à la longueur du jour }{ }^{2}\end{array}$} \\
\hline sur 1 mois & 0,6 & 0,5 & 0,7 & 0,8 & 0,8 \\
\hline sur $70-80 \mathrm{dj}$ & 0,5 & 0.5 & 0,5 & 0.8 & 0,9 \\
\hline
\end{tabular}

\footnotetext{
${ }^{1}$ Calculée en centièmes d'heure par jour sur une décade. 2 Critère de Cao et Moss (1989). Le nombre de jours pris en compte pour faire les 70 à 80 dj dans les 5 situations culturales est respectivement : $12,11,9,6,5$.
} 


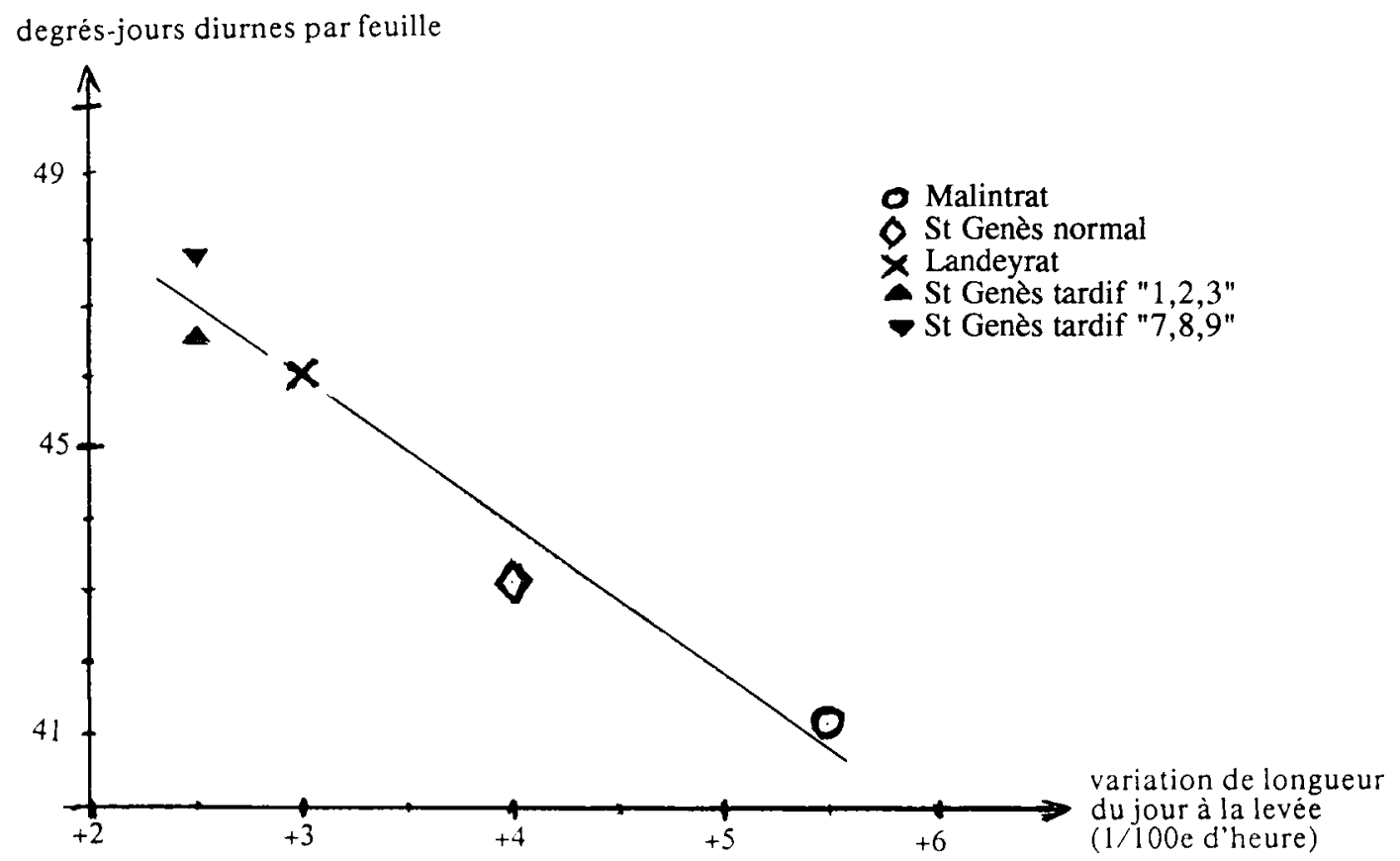

Fig 3. Phyllochrones photothermiques des plantes de type $\mathrm{T} 1+$ en conditions $\mathrm{BN}$, rapportés à la variation initiale de la longueur du jour. La droite de régression a pour équation : $y=52,2^{* *}-2,07^{* *} \times\left(r^{2}=95 \%{ }^{* *}\right) .^{* *}$ : significatif à $1 \%$.

d'émission des feuilles observées entre types de tallage sur blé d'hiver dans le Bassin parisien (Masle-Meynard et Sébillotte, 1981a), même s'il subsiste une variabilité résiduelle non négligeable (tableau $\mathrm{V}$ ). Le sens des différences significatives est le même quelles que soient les conditions et identique à celui constaté par les auteurs cités.

Le ralentissement de l'émission des feuilles par un manque d'azote ou par un semis dru (tableau VI) est généralement significatif malgré des fournitures d'azote probablement moins contrastées que celles obtenues par l'utilisation de solutions carencées (Dale et Wilson, 1978; Natr et Apel, 1983) et une gamme de densités plus réduite que celle de Kirby et Faris (1970). Les effets obtenus sont conformes aux constatations des auteurs cités. La différence d'écart BO-BN entre les 2 situations de Saint-Genès tardif suggère que, dans nos conditions, la compacité agit surtout en réduisant l'accès des racines à l'azote fourni par le sol. Par contre, l'effet densité ne peut se ramener à une simple concurrence entre plantes pour l'azote : les niveaux de nutrition (tableau III) ne diffèrent pas entre $\mathrm{BN}$ et FN dans les 3 situations où ces traitements présentent une différence significative de vitesse d'émission (tableau $\mathrm{VI}$ ). Une concurrence pour la lumière ne peut être exclue, mais elle devrait être modeste là où le couvert n'est qu'incomplètement fermé, c'est-à-dire en montagne.

L'absence d'effet significatif des conditions culturales sur l'émission des feuilles des céréales, généralement admise (par exemple par Kirby et al, 1985) sur la base de travaux sur blé d'hiver (Masle-Meynard et Sébillotte, 1981a), ne vaut pas pour l'orge de printemps. Les différences observables entre nos cultures d'altitude (fig 2) sont sans doute particulièrement marquées car elles cumulent des écarts significatifs entre plantes de même type et des changements de fréquence des types de tallage qui vont dans le même sens.

\section{Effets climatiques entre situations culturales}

Le raccourcissement du phyllochrone obtenu en retardant le semis d'une orge de printemps (Kirby et al, 1985) ne s'observe plus pour nos semis nettement postérieurs à l'équinoxe (tableau VII). Les résultats de Wright et Hugues (1987) révèlent le même phénomène. D'un autre côté, les différences de phyllochrone exprimé en temps photothermique sont inattendues après les conclusions de Masle et al (1989). II n'y a ce- 
pendant pas contradiction avec ces auteurs : nos dates de semis correspondent à celles qui, dans leur étude, s'écartent de la représentation générale.

Dans l'état actuel des modèles utilisables, nos résultats nécessitent d'admettre l'effet de conditions initiales sur l'émission des feuilles. Le critère de Cao et Moss permet de conserver une simple expression thermique du temps, mais sa validité dépend du choix d'une période de conditionnement assez longue. En outre, il existe des différences de coefficients de régression selon le lot de données (15 sur nos résultats, 30 ou 60 chez les auteurs); elles plaident contre sa signification biologique. La liaison du phyllochrone photothermique à la variation initiale de la photopériode (fig 3 ) introduit une redondance, mais elle a le même sens que celles qu'observent Kirby et al (1982) et Ellis et Russell (1984) pour le phyllochrone thermique chez l'orge. Les critères disponibles dans la littérature ne constituent sans doute que des manières de quantifier une même condition climatique. En analyse de climats naturels, on tend d'ailleurs à les employer indifféremment (Cao et Moss, 1991).

\section{Conditionnement initial ou modulation du phyllochrone par des facteurs contemporains?}

L'hypothèse d'un réglage précoce d'une horloge interne à chaque plante permet de concilier un allongement des organes très variable au cours du temps (Kirby, 1973; Kirby et al, 1982) avec la régularité du rythme de dégainage des feuilles successives et avec les différences de rythme entre milieux. Malvoisin (1984) a démontré l'existence d'un tel conditionnement initial en transférant en conditions contrôlées standard des plantes élevées en milieux naturels contrastés. Le conditionnement est acquis au plus tard lors du déroulement de la $5^{\mathrm{e}}$ feuille. II subsiste néanmoins toujours une certaine variabilité du rythme d'émission des feuilles sur une même tige (Hay et Kemp, 1990). Elle pourrait représenter une modulation par les variations de milieu contemporaines de l'émission de chaque feuille.

L'approche de Masle et al (1989) privilégie une modulation du phyllochrone par la longueur du jour contemporaine de l'apparition des feuilles. Nos résultats montrent que leur temps photothermique ne permet pas d'éviter dans tous les cas le recours au conditionnement des plantules par le climat en début de végétation, conditionnement admis par la plupart des auteurs (Baker et al, 1980; Kirby et al, 1982; Ellis et Russell, 1984; Cao et Moss, 1991).

Par leurs effets sur la croissance, les facteurs nutritionnels et la concurrence semblent pouvoir moduler au cours du temps le rythme d'émission des feuilles. Sur les talles, très sensibles aux pénuries (Masle, 1985), ce rythme ralentit effectivement avant l'arrêt total de croissance qui précède la mort de la tige (Davidson et Chevalier, 1990). Il reste cependant régulier et parallèle à celui des talles survivantes jusqu'à l'approche immédiate de l'arrêt, malgré des différences sans cesse accrues en taille et en gains de poids (Masle-Meynard, 1981). Sauf quand la pénurie devient létale, le phyllochrone apparaît peu sensible à la nutrition contemporaine. Sur les brins maîtres, des différences peuvent portant se manifester au champ avec un écart de nutrition azotée de seulement 15-20\% (fortes densités de Malintrat et basses densités à SaintGenès normal, voir tableaux VI et III). Si I'on observe que les différences obtenues par Dale et Wilson (1978) et Natr et Apel (1983) entre soIution complète et solution carencée l'ont été sur des plantules élevées dès le départ dans ces conditions, on pourrait rendre compte de ces comportements apparemment contradictoires en admettant un conditionnement des plantules par le niveau de la ressource en début de végétation.

Chez Kirby et Faris (1970), l'effet densité est observable dès le déroulement de la $4^{\mathrm{e}}$ feuille à 1600 pieds $/ \mathrm{m}^{2}$, mais avec une disposition très régulière du semis plaçant chaque plante à 2,5 $\mathrm{cm}$ de ses voisines. Des plantes à 4 feuilles étant en tout début de tallage (Masle-Meynard et Sébillotte, 1981b), une concurrence pour la lumière est alors peu probable. Nos fortes densités en lignes espacées réalisent une distance moyenne de $0,7-1,5 \mathrm{~cm}$ entre plantes sur la ligne, selon les situations. Plus qu'une concurrence nutritionnelle, on pourrait alors envisager un conditionnement initial par perception de la présence de plantes voisines: des réponses morphogénétiques très précoces à la qualité de la lumière réfléchie sont maintenant bien établies (Ballaré et al, 1987; Mancinelli, 1991).

\section{CONCLUSION}

Dans un peuplement donné d'orge de printemps, il existe des différences systématiques 
de vitesse d'émission des feuilles du brin maître selon le type de tallage des plantes. Pour des plantes de même type, la fourniture d'azote et la densité du peuplement modifient ce rythme d'émission au champ. En altitude, les écarts entre cultures sont accrus par leurs différences de composition en types de tallage à émission lente ou rapide des feuilles.

Pour des plantes de même type et des conditions nutritionnelles semblables entre lieux et dates de semis, les phyllochrones ne peuvent s'expliquer que par des conditions climatiques initiales. Un conditionnement initial pourrait être aussi induit par des facteurs nutritionnels ou la concurrence.

\section{REMERCIEMENTS}

Je remercie $P$ Pluchard et $J$ Masle pour leurs critiques constructives et leurs suggestions lors de la mise au point de cet article.

\section{RÉFÉRENCES}

Bachacou J, Masson JP, Millier C (1981) Manuel de la programmathèque statistique Amance 81. INRA, Champenoux

Baker CK, Gallagher JN, Monteith JL (1980) Daylength change and leaf appearance in winter wheat. Plant Cell Environ 3, 285-287

Baker JT, Pinter PJ, Reginato RJ, Kanemasu ET (1986) Effects of temperature on leaf appearance in spring and winter wheat cultivars. Agron $J 78$, 605-613

Ballaré CL, Sanchez RA, Scopel AL, Casal JJ, Ghersa CM (1987) Early detection of neighbour plants by phytochrome perception of spectral changes in reflected sunlight. Plant Cell Environ 10, 551-557

Boone MYL, Rickman RW, Whisler FD (1990) Leaf appearance rates of two winter wheat cultivars under high carbon dioxide conditions. Agron $J 82$, 718-724

Cannell RQ (1969) The tillering pattern in barley varieties. 1. Production, survival and contribution to yield by component tillers. J Agric Sci Camb 72, 405-422

Cao W, Moss DN (1989) Temperature and daylength interaction on phyllochron in wheat and barley. Crop Sci 29, 1046-1048

Cao W, Moss DN (1991) Phyllochron change in winter wheat with planting date and environmental changes. Agron J 83, 396-401

Dale JE, Wilson RG (1978) A comparison of leaf and ear development in barley cultivars as affected by nitrogen supply. J Agric Sci Camb 90, 503-508
Davidson DJ, Chevalier PM (1990) Preanthesis tiller mortality in spring wheat. Crop Sci 30, 832-836

Duru M, Langlet A (1988) Indice foliaire, structure du peuplement de tiges et biomasse des repousses d'une luzerne irriguée. Agronomie 8, 603-611

Ellis RP, Russel G (1984) Plant development and grain yield in spring and winter barley. $J$ Agric $S c i$ Camb 102, 85-95

Gallagher JN, Biscoe PV, Dennis-Jones R (1983) Environmental influences on the development, growth and yield of barley. In: Barley, production and marketing (GM Wright, RB Wynn-Williams, eds) Agron Soc New Zealand, 21-49

Gosse G, Varlet-Grancher C, Bonhomme R, Chartier M, Allirand JM, Lemaire G (1986) Production maximale de matière sèche et rayonnement solaire intercepté par un couvert végétal. Agronomie 6, 4756

Greenwood DJ, Neeteson JJ, Draycott A (1986) Quantitative relationships for the dependance of growth rate of arable crops on their nitrogen content, dry weight and aerial environment. Plant Soil 91, 281301

Haun JR (1973) Visual quantification of wheat development. Agron J 65, 116-119

Hay RKM, Delécolle R (1989) The setting of rates of development of wheat plants at crop emergence influence of the environment on rates of leaf appearance. Ann Appl Biol 115, 333-341

Hay RKM, Kemp DR (1990) Primordium initiation at the stem apex as the primary event controlling plant development: preliminary evidence from wheat for the regulation of leaf development. Plant Cell Environ 13, 1005-1008

Kirby EJM (1973) The control of leaf and ear size in barley. J Exp Bot 24, 567-578

Kirby EJM, Faris DG (1970) Plant population induced growth correlations in the barley plant main shoot and possible hormonal mechanisms. $J$ Exp Bot 21, 787-798

Kirby EJM, Appleyard M, Fellowes G (1982) Effect of sowing date on the temperature response of leaf emergence and leaf size in barley. Plant Cell Environ 5, 477-484

Kirby EJM, Appleyard M, Fellowes G (1985) Effect of sowing date and variety on main shoot leaf emergence and number of leaves of barley and wheat. Agronomie 5, 117-126

Klepper B, Belford RK, Rickman RW (1984) Root and shoot development in winter wheat. Agron $J 76$, 117-112

Kobold F (1974) Ertragsverhalten von Sommergerste und Winterweizen in Abhängigkeit von der Höhenlage. Akad Landwirtschaftswiss. Berlin, Tagungsber 127, 137-142

Lafarge M (1991) Une démarche d'étude agronomique des climats naturels; le cas de différences d'altitude. Agronomie 11, 369-381 
Malvoisin P (1984) Organogénèse et croissance du maître brin du blé tendre (Triticum aestivum) du semis à la floraison. 1. Relations observées entre la croissance foliaire et la différenciation des ébauches foliaires ou florales. Agronomie 4, 557564

Mancinelli AL (1991) Phytochrome-mediated detection of changes in reflected light. Plant Physiol 95, 144151

Masle-Meynard J (1981) Élaboration du nombre d'épis d'un peuplement de blé d'hiver en situation de compétition pour l'azote. 1. Mise en évidence d'un stade critique pour la montée d'une talle. Agronomie 1, 623-632

Masle-Meynard J (1982) Élaboration du nombre d'épis d'un peuplement de blé d'hiver en situation de compétition pour l'azote. 2. Modélisation du nombre d'épis. Agronomie 2, 17-24

Masle $J$ (1985) Competition among tillers in winter wheat: consequences for growth and development of the crop. In: Wheat growth and modelling (W Day, RK Atkin, eds) NATO ASI serie A, vol 86, Plenum Press, New York, 33-84
Masle-Meynard J, Sébillotte M (1981a) Étude de l'hétérogénéité d'un peuplement de blé d'hiver. 1. Notion de structure du peuplement. Agronomie 1, 207-216

Masle-Meynard J, Sébillotte M (1981b) Étude de I'hétérogénéité d'un peuplement de blé d'hiver. 2. Origine des différentes catégories d'individus du peuplement; éléments de description de sa structure. Agronomie 1, 217-224

Masle J, Doussinault G, Farquhar GD, Sun B (1989) Foliar stage in wheat correlates better to photothermal time than to thermal time. Plant Cell Environ $12,235-247$

Midmore DJ, Cartwright PM, Fischer RA (1984) Wheat in tropical environment. 2. Crop growth and grain yield. Field Crops Res 8, 207-227

Natr L, Apel P (1983) The effect of phosphorus and nitrogen deficiency on growth of seedlings of spring barley in dependance of irradiance: growth analysis. Biol Plant 25, 425-432

Ntonga J (1992) Déterminisme du nombre de grains par épi chez le blé d'hiver : influence des facteurs trophiques $(C, N)$. Thèse Univ Blaise Pascal, Clermont-Ferrand, $122 \mathrm{p}$ 